\title{
FLOATING-DISK PARYLENE MICROVALVE FOR SELF-REGULATING BIOMEDICAL FLOW CONTROLS
}

\author{
Po-Jui Chen ${ }^{1}$,Damien C. Rodger ${ }^{2}$, Mark S. Humayun ${ }^{2}$, and Yu-Chong Tai ${ }^{I}$ \\ ${ }^{1}$ California Institute of Technology, Pasadena, CA, USA \\ ${ }^{2}$ University of Southern California, Los Angeles, CA, USA
}

\begin{abstract}
A novel self-regulating parylene microvalve is presented in this paper with potential applications for biomedical flow controls. Featuring a free-floating bendable valve disk and two-level valve seat, this surface-micromachined polymeric valve accomplishes miniature pressure/flow rate regulation in a band-pass profile stand-alone without the need of power sources or active actuation. Experimental data of underwater testing results have successfully demonstrated that the microfabricated in-channel valve can regulate water flow at $0-80 \mathrm{mmHg}$ and $0-10 \mu \mathrm{L} / \mathrm{min}$ pressure/flow rate level, which is perfectly suitable for biomedical and lab-on-a-chip applications. For example, such biocompatible microvalve can be incorporated in ocular implants for control of eye fluid drainage to fulfill intraocular pressure (IOP) regulation in glaucoma patients.
\end{abstract}

\section{INTRODUCTION}

Flow controls enabled by passive microfluidic valves have great value in minimizing integration and operation complexity as well as manipulating fluids at microscale in integrated microsystems for biomedical and lab-on-a-chip applications [1]. With different valve paradigms, two flow regulation profiles as shown in Fig. 1 can be created for different purposes. Different from the "half-pass" flow profile which can be widely used in general flow regulators [2][3], the "band-pass" profile is more favorable to drug delivery and biofluid transportation in implantable medical microdevices because of its low-off and high-off threshold characteristics with safety considerations [4][5]. In physical intraocular pressure (IOP) regulation for glaucoma patients, for example, shunting eye fluid in a customized pressure band protects the eye both from hypotony when the IOP is lower than the expected value (e.g., $10 \mathrm{mmHg}$ ) and from excessive high-pressure high-flow-rate drainage when IOP acutely spikes (e.g., $50-80 \mathrm{mmHg}$ ) due to external interferences such as rubbing or pressing on the eye. These features enable safe and effective pressure/flow rate controls within a normal regulation range.

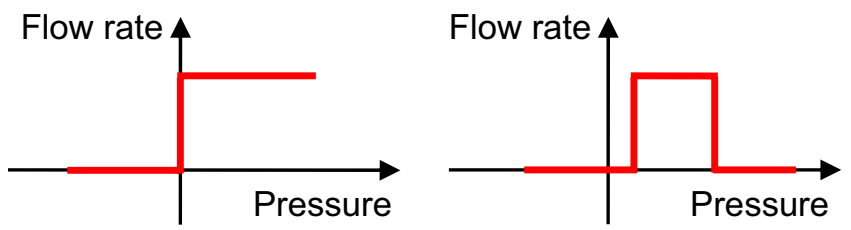

Figure 1. Typical flow regulation profiles: (left) "Half-pass" regulation, in which constant flow rate is maintained with forward pressure loading; (right) "Band-pass" regulation, in which the flow is conducted only within a pressure range.
Previously a dual-valved microfluidic device comprising a normally-closed and normally-open check valve was reported to realize flow control with the described band-pass profile [5]. In this work, derived from the recently reported nearly ideal floating-disk micro check valve [6], a single-valved device has been developed. With its exclusive self-regulating behavior, this alternative is designed to greatly reduce design/processing constraints while achieving identical functionality as the dual valves in a stand-alone fashion. By utilizing parylene as the essential biocompatible thin-film material, this proposed polymeric microvalve potentially has the capability of controlling biomedical flows in integrated implantable and lab-on-a-chip systems.

\section{DESIGN}

The design of the microvalve is illustrated in Fig. 2 incorporating an unclamped bendable "floating disk" and a two-level valve seat as the key elements to passively and mechanically achieve the self-regulating behavior. Being not connected or tethered to any anchor structures, this disk acts as a free plate in a simply supported condition confined in the space between valve seat and cap. This configuration enables immediate out-of-plane disk movement with minimal applied pressure loading. Accordingly, the fluidic pathway is closed once when the disk is pushed against the valve cap, resulting in the valve leakage-proof to flows in the backward direction. The forward flows are also blocked when the flexible floating disk is pushed against the valve seat under certain pressure loading. However, if a sufficient pressure is built up to overcome a predetermined threshold point a substantial deformation is then created in the disk with curved-up overhang, so the fluidic channels/trenches surrounding the edge of the disk on the top level of valve seat are exposed and flows can be conducted through them. This behavior defines the threshold pressure of the entire valve operation. On the other hand, the flow can also be blocked when the fluidic port on the bottom level of valve seat is closed by the highly bent disk with maximum deflection at center. This behavior defines the cut-off pressure of the entire valve operation. As a result, these combined effects fundamentally realize unpowered self-regulating band-pass operation of the microvalve. The polymer parylene $\mathrm{C}$ (poly-para-xylylene $\mathrm{C}$ ) is selected as the structural material because of its mechanical flexibility (Young's modulus $\sim 4$ $\mathrm{GPa}$ ), ease of processing, CMOS/MEMS processes compatibility, chemical inertness, and biocompatibility (FDA approved USP Class VI grade). All these characteristics favor the fabrication of flexible floating disk diaphragm and facilitate the microvalve compliant with medical and biological requirements. 

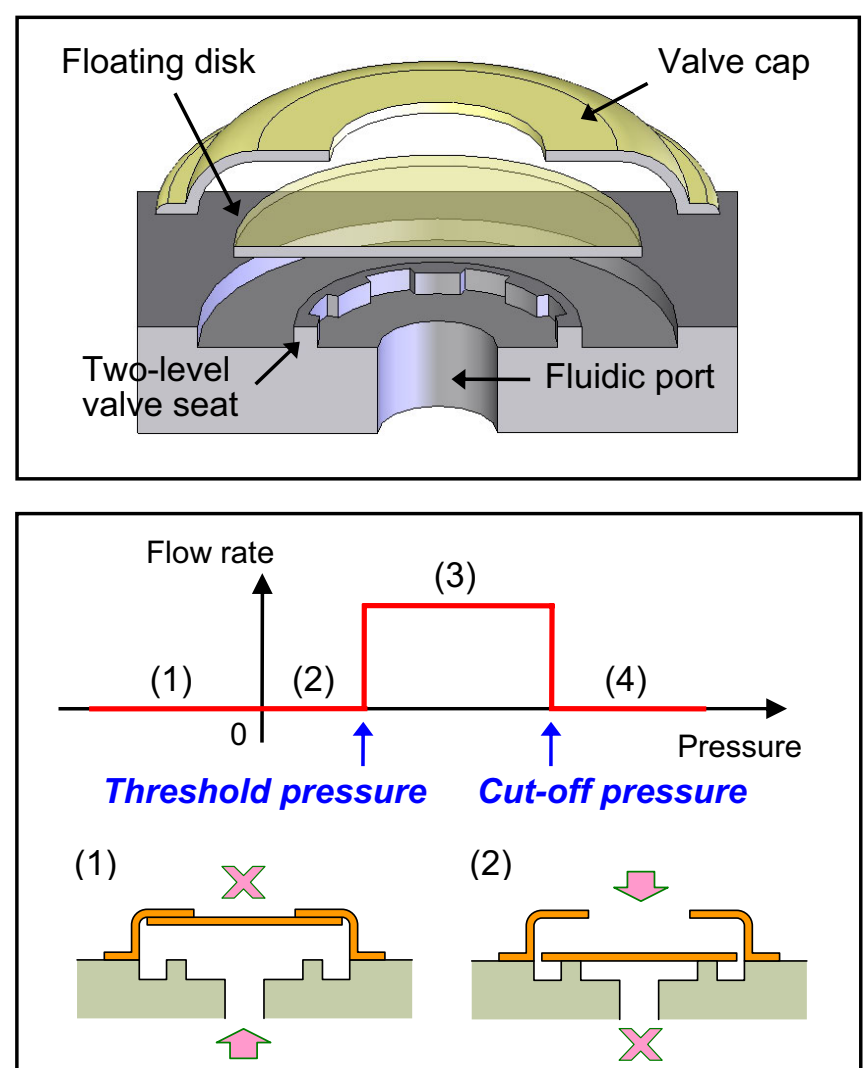

(3)

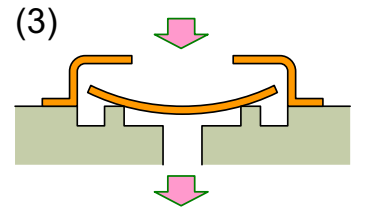

(4)

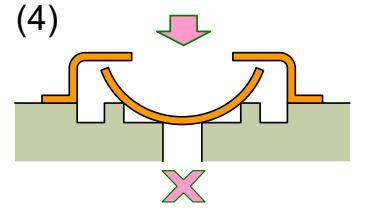

Figure 2. Microvalve design: (top) Conceptual schematic (exploded view); (bottom) Self-regulation mechanism. Arrows denote flow directions through the valve.

\section{FABRICATION}

Fig. 3 depicts the fabrication process started by thermally growing $2 \mu \mathrm{m}$ oxide on a double-side-polished wafer. The fluidic coupling ports were defined by performing oxide patterning and deep reactive-ion etching (DRIE) on the backside of wafer until an approximately $50-\mu \mathrm{m}$-thick silicon membrane was created. After that, another DRIE was used to form the two-level structures on the silicon valve seats. Using photoresist as a sacrificial material, a multi-layer polymer surface-micromachining technology was then exploited to form three parylene C structural layers as the disk diaphragm $(1 \mu \mathrm{m}$ thick $)$, the valve cap (5 $\mu \mathrm{m}$ thick), and the fluidic channel ( $10 \mu \mathrm{m}$ thick) of the microvalve. Parylene patterning was performed using oxygen plasma etching with photoresist as the mask material. During the process the sacrificial photoresist was hard-baked at $120^{\circ} \mathrm{C}$ if applicable for edge-smoothening and degassing purposes. Given that the wafer surface was not flat with two-level silicon seat structures, planarization of the first layer sacrificial photoresist coating required more careful calibration to guarantee the flatness of the free-plate disk diaphragm for reliable valve operation. Other than that, all deposited parylene $\mathrm{C}$ layers were roughened using a short duration of oxygen plasma treatment to prospectively reduce stiction effects when operating device. In order to enhance the pressure loading capacity of the device, gas-phase $\mathrm{XeF}_{2}$ silicon roughening was performed before the second and third parylene layer coating to promote adhesion of the valve cap and the fluidic channel connected to substrate. Finally, the remaining silicon membrane on fluidic port was etched away from the backside of the wafer to create through-wafer holes thus enabling not only sacrificial photoresist stripping with acetone but fluidic access for device operation. The released devices were supposed to be kept in liquid solutions after fabrication to prevent stiction of the disk diaphragm due to lack of supporting structures during drying. However, a pneumatic disk-popping process can still be exploited to rejuvenate the device if air operation is needed [7].

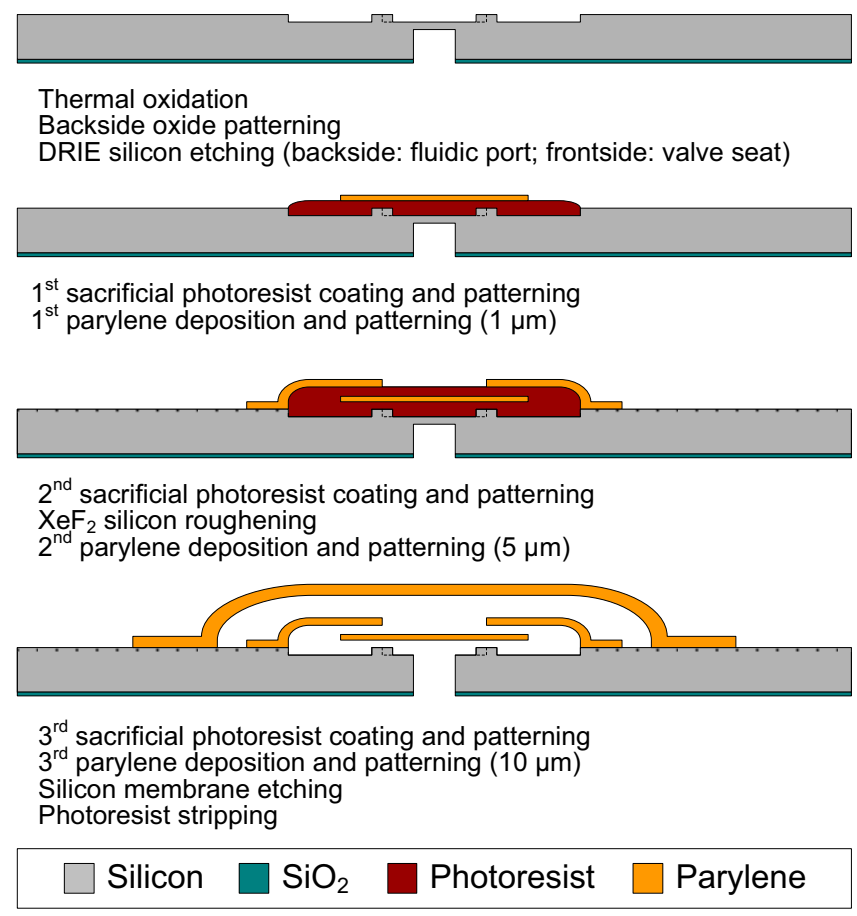

Figure 3. Fabrication process flow.

Two-level silicon valve seats with two different circular shape designs as shown in Fig. 4 were implemented to study their influence on (1) boundary conditions on the floating disk and (2) overall fluidic conductance. By having different numbers and dimensions of radiating trenches, the valve seats can possibly have different supporting points for the flexible parylene disk when they are in contact, resulting in different equivalent pressurized area of the disk and associated disk overhang. Therefore, the boundary conditions of the microvalve can be altered to have different disk deformations with different resultant threshold pressure points as indication. Theoretically, the overall fluidic resistance generated from the valve seat channels/trenches is also slightly different in the two different contours under the same pressure loading because the disk is deformed to a 
different extent, which induces different exposure of the valve seat channels to fluidic pathways. Nevertheless, this fluidic resistance change is believed to be insignificant, especially for miniature pressure/flow rate controls. 3-D surface profile scans of the valve seats show that the extrusion height was controlled to be approximately $5 \mu \mathrm{m}$ in order to obtain the predetermined high-off characteristic for band-pass flow regulation of interest with the given device dimensions. In addition, it was also feasible to cover this step height with relatively planarized photoresist at the end. The rim width of the valve seat extrusion should be as small as possible for better realization of simply supported condition to the disk diaphragm. Because photoresist was used as the sacrificial material in this work, the diameter of the patterned parylene disk needs to be smaller than that of the sacrificial photoresist base to avoid the relatively rounded/bumpy edge of photoresist after baking. In this way the disk can maintain its flatness in the peripheral region while increasing the planar clearance of the valve disk/seat/cap combination. This fact brings up concerns about the tolerance of in-plane disk movements in the confines of the valve seat and cap so as need to be addressed for reliable valve performance in practice. Fig. 5 shows micrographs of the submerged fabricated in-channel floating-disk microvalves.
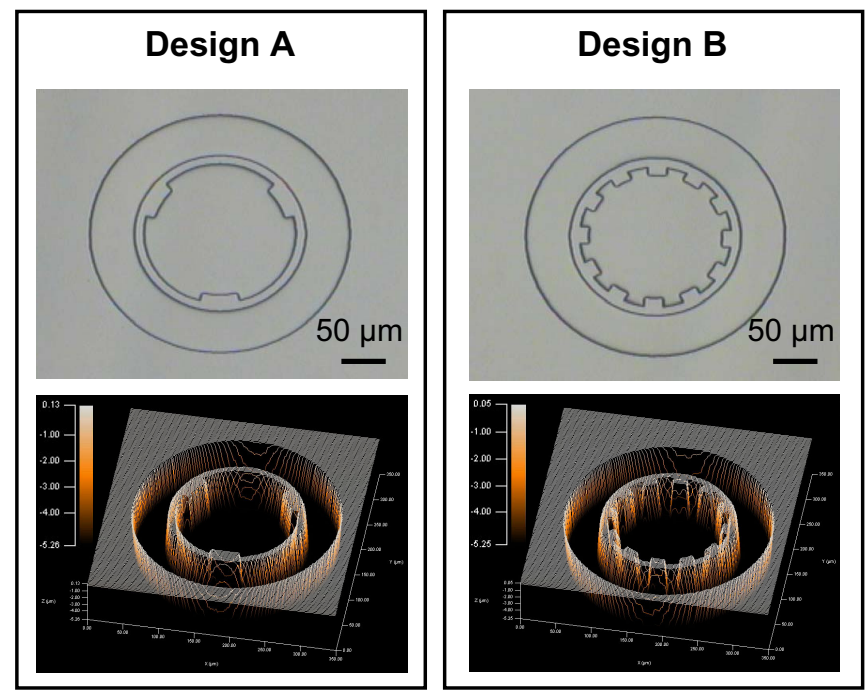

Figure 4. Micrographs and 3-D surface profile scans of two-level silicon valve seat with different designs.
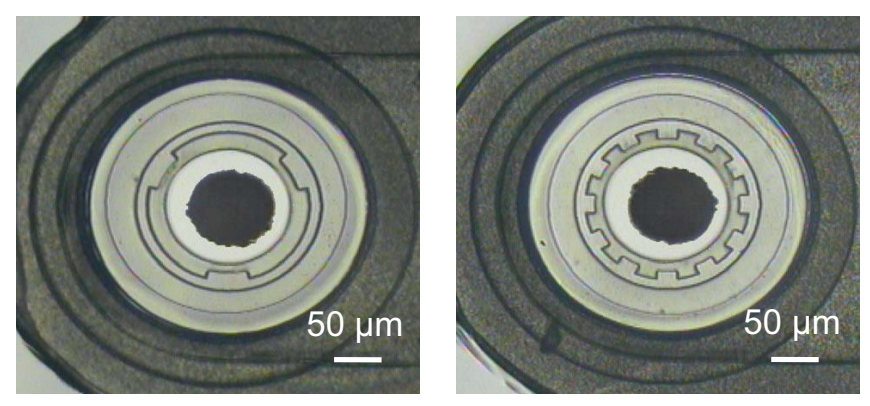

Figure 5. Micrographs of in-channel parylene valves (left: Design A; right: Design B) immersed in water (top view).

\section{TESTING AND RESULTS}

Device characterization was conducted using a customized testing setup as illustrated in Fig. 6 with the fabricated in-channel microvalves immersed in water. World-to-chip interfacing was accomplished by packaging the diced valved chip to a PEEK (poly-ether-ether-ketone) jig connected with regular tubing and fittings. Using water as the working fluid, microflows were introduced to the devices through their backside fluidic access ports with a combination of an off-chip pressure regulator and pressure gauge in control of the injection pressures. Flow rate measurement was performed by recording the marching velocity of the water column front in the capillary tube at back end before fluid collection. Air bubbles inside the system were completely removed prior to valve characterization so that the microvalves were ensured to be tested in a liquid environment.

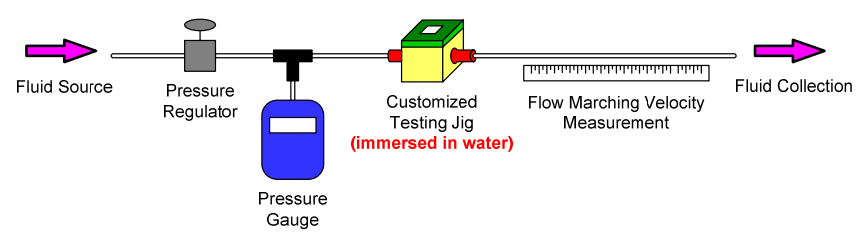

Figure 6. Schematic of fluidic setup for device testing.

Pressure responses of the microvalves were first tested to investigate their threshold and cut-off pressures with different valve seat designs. Testing data summarized in Table I show that the pressures points were within a range suitable for intraocular and most other biomedical applications $(0-80 \mathrm{mmHg})$ in good agreement with design expectations. Although different in valve seat geometry, microvalves of both designs obtained similar threshold pressure ( $\sim 10-20 \mathrm{mmHg})$ and cut-off pressure ( 65-75 $\mathrm{mmHg}$ ), which indicates that the difference of resultant performance is not significant as it was unobserved using the testing setup. In fact, the difference is believed to be more likely concealed by the variation of results caused by imperfect alignment between valve disk and seat during operations because of uncontrollable in-plane disk movement. It is also known that the double-side pattern alignment on the wafer can affect the final cut-off pressure values as the fluidic port would be off center from the designed disk diaphragm location, causing larger pressure loadings to seal the fluidic port and block the flow. All these factors can change the boundary conditions of the bendable disk and are worthy of further studies.

Table I Pressure testing results of fabricated microvalves

\begin{tabular}{lcc}
\hline & Design A & Design B \\
\hline $\begin{array}{l}\text { Threshold } \\
\text { pressure }\end{array}$ & $0.31 \pm 0.09 \mathrm{psi}$ & $0.23 \pm 0.04 \mathrm{psi}$ \\
Cut-off & $15.9 \pm 4.7 \mathrm{mmHg})$ & $(11.6 \pm 1.9 \mathrm{mmHg})$ \\
pressure & $(68.6 \pm 3.3 \mathrm{mmHg})$ & $(71.8 \pm 4.2 \mathrm{~mm} \mathrm{mmg})$ \\
\hline
\end{tabular}

Representation format: average \pm standard deviation $(n=5)$ 
The pressure/flow rate behavior of the in-channel microvalve was also characterized. A sample test result as shown in Fig. 7 demonstrated a passively controlled microflow having $0-100 \mathrm{mmHg}$ and $0-10 \mu \mathrm{L} / \mathrm{min}$ band-pass regulation ranges with the fabricated channel dimensions. Valves of both designs had similar resultant regulation performance. It is evident in the plot that both low-off and high-off characteristics were successfully achieved in experiments with no observable water flow below the threshold pressure point and greatly reduced flow rate (below $1 \mu \mathrm{L} / \mathrm{min}$ ) beyond the cut-off pressure point. In backward flow injection, as expected, no reverse leakage was observed with pressure loadings higher than 30 psi. Future work includes fluidic-structural-coupled device analysis and improvement of post-fabrication valve disk/seat/cap alignment in order to have a more quantitative understanding of the floating-disk microvalve behaviors.

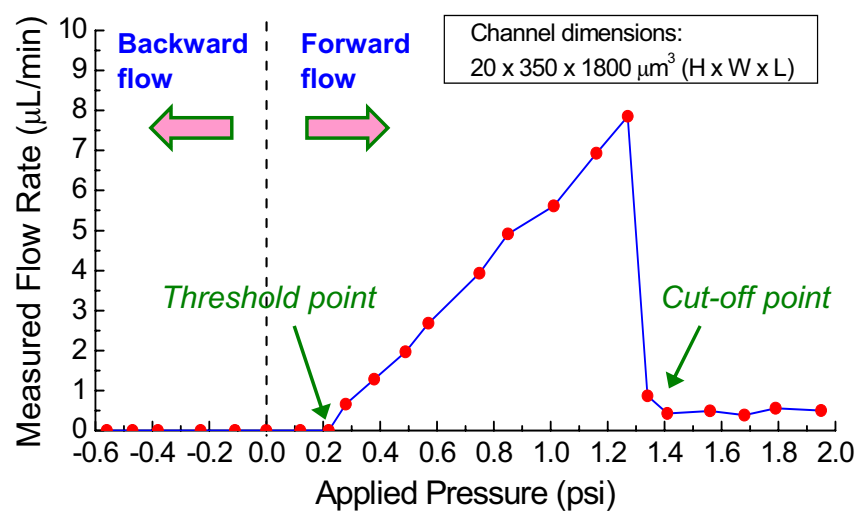

Figure 7. Pressurelflow rate testing of the in-channel valve (Design B) showing water flow control with expected regulation profile.

\section{CONCLUSION}

A parylene-based self-regulating floating-disk microvalve has been successfully developed. Its bendable free-plate disk diaphragm in association with a two-level valve seat accomplishes a specific band-pass flow profile without the need for active control components. A multi-layer polymer surface-micromachining technology using parylene $\mathrm{C}$ as the structural material was utilized to fabricate the in-channel microvalves with different valve seat geometries. Device tested in a water environment demonstrated excellent low-off and high-off characteristics below $\sim 10-20 \mathrm{mmHg}$ and above $\sim 65-75 \mathrm{mmHg}$ pressures, respectively, along with an achieved $0-10 \mu \mathrm{L} / \mathrm{min}$ flow rate range in the entire flow regulation regime. Supported by experimental results of the fluidic shunting capability and the unique biocompatible property of parylene, this microvalve is promising as a key microfluidic component to be integrated into systems in a wide variety of implantable and lab-on-a-chip paradigms for self-regulating biomedical microflow controls, such as eye fluid drainage for physical IOP regulation for glaucoma patients and biofluid transportations in other applications.

\section{ACKNOWLEDGEMENTS}

This work was supported in part by the Engineering Research Centers Program of the National Science Foundation under NSF Award Number EEC-0310723. The authors would also like to thank Mr. Trevor Roper for his fabrication assistance.

\section{REFERENCES}

[1] K.W. Oh and C.H. Ahn, "A Review of Microvalves," Journal of Micromechanics and Microengineering, 16(5), pp. R13-R39, 2006.

[2] J. Collier, D. Wroblewski, and T. Bifano, "Development of a Rapid-Response Flow-Control System Using MEMS Microvalve Arrays," Journal of Microelectromechanical Systems, 13(6), pp. 912-922, 2004.

[3] B. Yang and Q. Lin, "A Planar Compliance-Based Self-Adaptive Microfluidic Variable Resistor," Journal of Microelectromechanical Systems, 16 (2), pp. 411-419, 2007.

[4] P. Cousseau, R. Hirschi, B. Frehner, S. Gamper, and D. Maillefer, "Improved Micro-Flow Regulator for Drug Delivery Systems," Proc. MEMS 2001 Conference, Interlaken, Switzerland, Jan. 21-25, 2001, pp. 527-530.

[5] P.-J. Chen, D.C. Rodger, E. Meng, M.S. Humayun, and Y.-C. Tai, "Surface-Micromachined Parylene Dual Valves for On-Chip Unpowered Microflow Regulation," Journal of Microelectromechanical Systems, 16 (2), pp. 223-231, 2007.

[6] P.-J. Chen and Y.-C. Tai, "Floating-Disk Parylene Micro Check Valve," Proc. MEMS 2007 Conference, Kobe, Japan, Jan. 21-25, 2007, pp. 453-456.

[7] J. Xie, X. Yang, X. Q. Wang, and Y.-C. Tai, "Surface Micromachined Leakage Proof Parylene Check Valve," Proc. MEMS 2001 Conference, Interlaken, Switzerland, Jan. 21-25, 2001, pp. 539-542. 